\title{
La quimera del euro-. \\ Acerca de algunos elementos compositivos \\ en español
}

\author{
Joaquín García Palacios \\ Universidad de Salamanca
}

La prensa diaria de los últimos años ha ido proporcionándonos con sus titulares una buena cantidad de posibles encabezamientos para la investigación que estábamos llevando a cabo ${ }^{1}$. Bien es verdad que en todos esos casos los periodistas se estaban moviendo por los dominios de la economía o la política, y nuestro interés -lingüístico- residía únicamente en ese segmento de palabra que ha ido convirtiéndose en asiduo visitante de los periódicos españoles al convivir con comicios, diputados, escépticos u optimistas, o, sobre todo, al pasar a ser la denominación oficial de la moneda única europea.

A pesar de la innegable relación de esa unidad léxica que ya circula con plena autonomía por las distintas lenguas europeas con este formante $^{2}$, los límites de este trabajo dejan fuera a esa unidad, pues quere-

1 Me costó descartar el título del anuncio institucional (Ministerio de Economía y Hacienda español, y Unión Europea) "El qué, dónde, cuándo, cómo y porqué del euro" (28.x.97), porque reúne muchas preguntas, casi tantas como a las que pretendo contestar en este artículo, pero al final preferí aludir a lo que esa secuencia tiene de enigmático y conflictivo, y tomé el título prestado y ligeramente modificado de otro artículo con resonancias cinematográficas cuyo tema principal también era la moneda única europea (Enrique Barón Crespo "La quimera del euro", El País, 12.10.96, p. 11).

2 La cercanía del acontecimiento facilita considerablemente el seguimiento de la pequeña historia de la gestación de esta voz, que vemos reflejada en algunos comentarios que aparecieron en aquellos días de diciembre de 1995 acerca de las deliberaciones en la cumbre de Madrid para poner nombre a la nueva moneda: "González 
mos centrarnos únicamente en los distintos aspectos que encierran en sí las formaciones -sencillas, al menos en apariencia- que se han producido mediante la adición del segmento euro- en posición prefijal a una palabra ya existente. Y vamos a hacerlo considerando la realidad que nos muestran los textos en los que aparece de manera natural esa secuencia, el estado actual de la bibliografía que se ha preocupado de estudiar este asunto y las apreciaciones deducibles de las obras lexicográficas en que se halla recogida. Todo ello con la intención de contribuir a aclarar los problemas conceptuales que están en la base del caos terminológico existente en la actualidad para referirse a ese elemento y a las palabras formadas con él.

\section{PUNTOS DE PARTIDA}

El seguimiento continuo de la prensa española a lo largo de los últimos seis años nos ha permitido observar que la timidez con que hacía su aparición alguna unidad léxica con el formante euro-, se ha convertido en los últimos tres años casi en frecuencia descarada ${ }^{3}$. Esa frecuencia alcanza los límites de la cotidianeidad si ensanchamos nuestra búsqueda y, además de rastrear las voces que poseen ese segmento, indagamos en todos esos casos en los que se generan nuevas unidades

propuso de entrada el nombre de euro, sin añadidos (nada de euromarco o europeseta, como acariciaba inicialmente el gobierno de Bonn), que es el mismo en todas las lenguas de la UE, es simple y simboliza a Europa" (El País, 16.12.95). Comentarios que incidieron como era de esperar en consideraciones lingüísticas: "La moneda europea se llamará Euro. Euro será su nombre completo y no un prefijo que se anteponga a las monedas nacionales" (El País, 17.12.95). El belga Jean Luc Dehaene, enfocaba el asunto desde otra perspectiva: "Creo que el nombre euro puede suscitarlo [el consenso], sin necesidad de añadirle un sufijo que recuerde a las antiguas monedas nacionales" (El País, 18.12.95). No salía tampoco de lo lingüístico el periódico Times de Londres cuando ironizaba sobre la pronunciación de la nueva palabra: “Oihro, eoorro, ero, evro... jese era el gran consenso!" (El País, 18.12.95).

3 La mirada alerta nos ha llevado a no dejar pasar sin ser registradas previamente otras voces con las que a lo largo de este tiempo nos hemos topado en otros medios informativos (fundamentalmente radio y televisión) o en los lugares más diversos, tanto en España como en otros países europeos. Aunque estas apariciones solían ser ocasionales, sin llegar en ningún momento a tener la relevancia que los usos de la prensa escrita. 
siguiendo un procedimiento igual o similar al que se da en algunas de las formaciones con euro-.

A nada perspicaces que seamos vemos una cercanía entre el fenómeno que se produce en ese caso y el que se da en otros con formantes como narco- o petro-. De hecho, aunque consideremos como relativamente nuevas a las formaciones con euro-, no podemos pensar en el nacimiento de un nuevo procedimiento de formación de palabras en español pues, aunque éste sólo haya comenzado a ser verdaderamente productivo en los últimos años ${ }^{4}$, sus orígenes hay que buscarlos mucho antes, al producirse la formación de unidades con el añadido en posición prefijal de los formantes tele- o radio- a una base de composición ${ }^{5}$. El mecanismo utilizado para la formación de esas nuevas palabras había sido el mismo que con euro-: pérdida de la segunda parte de una palabra, normalmente de origen culto, con lo que queda un segmento bisílabo que se antepone a una palabra preexistente. Es lo que había sucedido con petro- (de petróleo) o narco- (de narcótico), unidos a dólar en petrodólar o narcodólar, o con tele- (de televisión), añadido a adicto en teleadicto.

En todos esos casos nos encontramos ante un elemento nuevo, propio de las lenguas actuales que, aunque sea homógrafo del formante culto, no puede ser confundido con él, ya que se trata de un elemento surgido del acortamiento de la forma patrimonial, compuesta, que sí que incluía el tema de composición de origen culto. Con lo cual tendríamos que hablar de la homonimia que se produce: la misma forma narco- es de origen culto y significa 'sopor, modorra' en narcótico, pero procede del truncamiento de la forma patrimonial narcótico, con el significado de 'droga' en narcodólar, narcopresidente o narcoestado ${ }^{6}$.

4 Sobre la noción de productividad y su relación con la formación de palabras, véase el estudio de Teresa Vallès (1996).

5 Hace ya mucho tiempo que en español se estudiaron las formaciones con tele- (Romero Gualda, 1976), analizadas diez años antes para el francés (Peytard, 1964). Radio- fue estudiado por Pérez Lagos (1985).

6 De la misma manera podemos hablar de petro1- (culto 'piedra') y petro2- (patrimo- 
Lo que en apariencia parece tan sencillo y equiparable a los múltiples casos en que una forma de palabra adquiere nuevos significados con el paso del tiempo, se complica cuando de lo que tratamos no es de una unidad léxica sino de un elemento inferior a ella, y cuando, además, nos situamos en una zona que se halla a caballo entre la composición y la derivación.

Los diccionarios no nos proporcionan demasiada ayuda para la solución de esos problemas. Hasta hace muy poco apenas recogían formas con euro-, y cuando lo hacían todas ellas se percibían como un conjunto sin fisuras cuando en realidad se trataba de varios grupos que formalmente coincidían en la posesión de ese segmento inicial.

Lo que sí nos indican los múltiples ejemplos que han aparecido en las obras lexicográficas en los últimos años es la relativa novedad de esta formación, y al mismo tiempo la vitalidad de un proceso que se halla en plena expansión?

Tampoco parece que nos encontremos ante un asunto que haya sido suficientemente estudiado.

nial 'petróleo'), tele1- (culto 'a distancia') y tele2- (patrimonial 'televisión'), foto1(culto 'luz') y foto2- (patrimonial 'fotografía'), dino1- (culto 'terrible') y dino2- (patrimonial 'dinosaurio'), auto 1- (culto 'por uno mismo') y auto2- (patrimonial 'coche'), etc. Ninguno de los casos anteriores está contemplado en su justa medida en la última edición del diccionario académico ( $21^{\mathrm{a}}$ ed., 1992).

7 Es muy significativo al respecto el caso de uno de los diccionarios de lengua más famosos para el español, el Diccionario de Uso del Español (DuE) de María Moliner. En su primera edición (1966) y en las múltiples reimpresiones posteriores únicamente se recoge el nombre del viento euro y unos cuantos derivados de Europa. La variación que se produce en la $2^{\mathrm{a}}$ edición (1998) constituye un buen reflejo de lo que ha cambiado la situación respecto a este elemento: se recogen dos entradas, euro 1 ('viento') y euro 2 ('moneda europea'), el "elemento prefijo" euro- con dos acepciones distintas, y a continuación las entradas euroasiático, eurocheque, eurocomunismo, eurocomunista, eurodiputado, eurodólar, euromisil y eurotúnel.

El menor tiempo transcurrido entre las dos últimas ediciones del DrAE (1984 y 1992) hace que las diferencias no sean tan grandes: únicamente el nombre del viento y la voz euroasiático en 1984. Ocho años después se reserva una entrada para euro- como "elemento compositivo" que aparece junto a euroasiático, eurocomunismo, eurocomunista, eurodiputado, eurodivisa y eurovisión. 
En 1993 la editora de un excelente libro sobre la formación de palabras en español reconocía la necesidad de "estudiar de manera más pormenorizada la barrera entre formas compuestas propias y aquellas dotadas de un primer elemento acortado o un alomorfo de tipo culto, de posible identificación con un prefijo". Es muy probable que Soledad Varela (1993: 15) al hacer esa sugerencia estuviera pensando en formas como euro- o alguna otra de las que acabo de señalar; al mismo tiempo observaba la necesidad de fijar los límites y diferencias entre unas y otras formas de composición, y de realizar estudios que caracterizasen convenientemente a esos formantes y a los procedimientos en los que éstos aparecían.

La situación en aquellos momentos era muy parecida a la que hoy nos encontramos. Las aportaciones que se han hecho hasta ahora, centradas en el estudio de algún caso concreto, han tenido un carácter eminentemente descriptivo con lo que, a pesar de su indudable valor, no han conseguido ofrecernos los elementos necesarios para la correcta comprensión del fenómeno que estamos tratando ${ }^{8}$. Y eso que éste no constituye un caso aislado en la morfología del español sino que, como ya hemos indicado, está íntimamente ligado a otros similares en los que se activa el mismo procedimiento aunque con distintos formantes.

Un fenómeno que, al mismo tiempo, adquiere una dimensión plurilingüe, y que quizás tendríamos que situar como una manifestación más de la globalización que nos invade, del mimetismo existente entre distintas manifestaciones lingüísticas de una misma cultura. Desde ese punto de vista puede que sea secundario saber si los primeros compuestos con euro- se dieron en español o en otra lengua (francés o inglés,

8 Sirva como ejemplo el artículo de Pérez Lagos sobre radio- donde explícitamente reconoce que sólo quiere reflejar una situación, y que no pretende "sacar conclusiones ni dar afirmaciones taxativas" (1985, p. 94). Descriptivos son también el artículo de Romero Gualda (1976) sobre tele- o el de Alvar Ezquerra (1978) sobre foto-, ambos en la línea marcada por Peytard (1964) en su artículo acerca del formante francés téle-. Otra es la línea de V. Alba de Diego (1983 y 1985), pero sus artículos tampoco pueden profundizar lo suficiente en el asunto que nos interesa al ser demasiado generales. 
como casi con toda seguridad ocurrió), y de allí se generalizó su uso al tratarse de unas formaciones que encajaban perfectamente con un procedimiento de formación de palabras ya existente en cada una de las lenguas que lo adoptaron y en las que no constituyó únicamente un préstamo aislado, sino una formación que comenzó a ser productiva. Quizá lo verdaderamente importante, independientemente de su origen, sea ver que se trata en efecto de un procedimiento ante el que cualquier lengua románica o el inglés pueden ser permisivos en su adopción, pues no resulta en absoluto extraño a sus sistemas de formación de palabras 9 .

Es importante también considerar la abundancia de denominaciones con que se alude al formante euro- y semejantes, así como al procedimiento que rige su adjunción a determinadas palabras. Y no lo es por un "capricho denominativo", sino por los problemas conceptuales que suelen esconder las cuestiones terminológicas cuando se encuentran envueltas en la confusión. Si en unos casos se habla de prefijoides o pseudoprefijos y en otros de elementos compositivos, no es debido a preferencias estilísticas, sino a que el fenómeno se halla a caballo entre la prefijación y la composición. De la misma manera que puede estar justificado el que en esos casos algunos autores acerquen el fenómeno a la composición culta y otros lo hagan a la patrimonial, el que unos hablen de reglas de formación de palabras y otros de analogía para la creación de las nuevas unidades.

Entraña dificultad encontrar un término preciso para aludir a los conceptos que ahora nos interesan, por lo que procederemos a separar y clasificar convenientemente los distintos ejemplos encontrados, así como a intentar aclarar las cuestiones conceptuales (morfológicas y semánticas) para de esa manera estar en condiciones de abandonar la denominación y el concepto de "pseudoprefijo", un "concepto incómodo", en palabras de Margarita Correia. Trataremos para ello de situarlo conceptualmente

9 Ha sido por tanto completamente natural la ayuda que han prestado a este trabajo los estudios que tratan este asunto en lenguas como el portugués, el francés o el catalán, así como las consideraciones de L. Bauer (1983) o B. Warren (1990) sobre la importancia de las combining forms en inglés. 
en el lugar adecuado, y enfrentarnos después con el establecimiento de unas denominaciones coherentes, motivadas, que no olviden los principios más elementales de la teoría terminológica.

\section{EL ENGAÑO DE LAS APARIENCIAS}

Viene siendo habitual tanto en obras lexicográficas como en artículos científicos la consideración conjunta de todas las formaciones en que aparece euro-. Sin embargo, la coincidencia formal no puede llevarnos a otorgar visos de homogeneidad a lo que esconde al menos tres subgrupos diferentes:

1. Compuestos del tipo euroasiático en los que se produce una relación de coordinación entre sus constituyentes, y en los que euroes un alomorfo del adjetivo europeo cuando éste tiene que aparecer en posición inicial del compuesto. En ellos, es la relación de precedencia de uno de los componentes la que únicamente puede hacer pensar en su mayor importancia sobre el que aparece en segundo lugar, pues en realidad, según su contenido, debería ser posible el intercambio posicional sin que se viera alterado el significado global del compuesto. Sin embargo, podemos observar tanto en éste como en otros casos similares que la posición inicial condiciona la aparición de determinado alomorfo: euroasiático vs. asiáticoeuropeo, euromagrebí o euromediterránea vs. jordano-europea ${ }^{10}$. Estamos así ante la que va a ser una de las características fundamentales de euro-, tanto en los compuestos de

10 En el último caso europeo adoptaría distintos alomorfos según la posición, algo que no ocurriría con jordano (eurojordano vs. jordanoeuropeo). Lo más normal, no obstante, es que se produzca la variación dependiendo de la posición: kosovoalbaneses vs. albanokosovares (referencias por desgracia demasiado frecuentes en todos los medios informativos entre los meses de marzo y junio de 1999), germanofrancés vs. franco-alemán ("Desafío franco-alemán a España en la UE", El País, 21.2.99). Observamos también cómo podemos hablar de una reunión hispano-lusa o hispano-portuguesa indistintamente, igual que la reunión puede ser luso-española o luso-hispana. En ambos casos son imposibles otras combinaciones que pudiéramos imaginar (*española-portuguesa, ${ }^{*}$ españolo-lusa, ${ }^{*}$ portugueso-hispana, etc.). 
este grupo como en los de otros: su especialización para la posición inicial de palabra.

En este grupo de compuestos se observa vacilación en cuanto a la utilización del guión, un signo que refleja una estructura de coordinación en la que se quiere incidir en que los dos miembros están al mismo nivel ${ }^{11}$.

Estos compuestos son las formaciones más antiguas con euro-. A pesar de ello, se trata de un procedimiento que continúa siendo productivo en la actualidad para dar cuenta de esa relación de coordinación con otro adjetivo de lugar o procedencia.

2. Compuestos subordinantes que surgen como fruto de un procedimiento de acronimia' ${ }^{12}$. El ejemplo más claro es Eurovisión (europea + televisión), un compuesto bastante antiguo (data de 1954 según el Dictionnaire des mots contemporains de P. Gilbert). En la misma línea puede ser considerada otra voz de la misma década o de la siguiente, eurócrata, interpretable como acrónimo de euro(peo) + (buró) crata, aunque quizá no habría que descartar alguna otra interpretación ${ }^{13}$.

11 Es loable el intento de simplificación del fenómeno para su mejor clasificación que se lleva a cabo en el Esbozo de una nueva gramática de la lengua española (1973: 152), y que posteriormente acepta M. Alvar Ezquerra (1995: 27). Se quedan, sin embargo, un tanto cortos, ya que la utilización del guión es en estos casos mucho más compleja de lo que ahí se señala. También mucho más anárquica. La vacilación en su uso lleva a que sin un orden lógico (lo normal en otros casos es que en cuanto avanza la lexicalización el guión del compuesto desaparezca) el guión aparezca o desaparezca caprichosamente: "La Conferencia Euro-Mediterránea de Ciudades Sostenibles arrancó ayer en Sevilla" (El País, 22.1.99). Cuatro años antes, según puede observarse en el ejemplo de la nota 29, el compuesto aparecía en el mismo periódico sin guión. La nueva Ortografía de la Lengua Española (1999: 83) opta por prescribir la escritura sin guión de los compuestos que "se sienten como consolidados", mientras que si no son sentidos como unidad el uso es potestativo.

12 Nos distanciamos del concepto de acronimia de Casado Velarde (1999: 5085) por considerarlo excesivamente abarcador. En su concepción todos los ejemplos con euro- que estudiamos serían casos de acronimia.

13 Podría tratarse también de un caso de composición a la manera culta en que se unen 
A pesar de que el procedimiento que da lugar a estas formaciones es poco productivo en la actualidad, podemos encontrar algunos casos de reciente aparición como el fr. eurolecte o el es. eurolalia, que nos están poniendo ante casos extremos para los que quizás tendríamos que hablar plenamente de composición a la manera culta, ya que la parte final del compuesto es sin duda un tema de composición culto, que se une a ese euro- que hace las veces igualmente de tema de composición, y que tampoco puede funcionar de manera autónoma en la lengua. En estos casos (entre los que nunca podríamos incluir eurovisión) euro- no remite a Europa sino a la Comunidad Económica Europea, hoy Unión Europea. La escasa productividad del fenómeno quizá se vea respaldada en el hecho de que estos dos últimos casos han sido creados con una buena dosis de humor. ${ }^{14}$

En cualquier caso, la estructura de estos compuestos, tanto si optamos por la acronimia como por la composición a la manera culta, es la misma que la de los compuestos cultos: subordinante, con el núcleo a la derecha.

3. Casos en los que un elemento se añade en posición inicial a una unidad libre y preexistente. En todos ellos, aunque admitan distintas formas para ser parafraseados, puede adivinarse una estructura subordinante del tipo complemento+núcleo, dando cuenta de una

los temas euro- y -crata ('que tiene el poder'). Su significado sin embargo nos lleva a inclinarnos por su interpretación como acrónimo, tal y como hace M. Correia (1989) para la voz portuguesa: 'burócrata europeo/de la UE').

14 Con humor interpretamos también el titular "Alicia en Eurolandia" que el autor de un sesudo artículo sobre economía utiliza para generar un sinónimo de la Unión Europea (Javier Gúrpide, El País, 1.3.99). A esta voz se refiere también un artículo anterior (El País, 15.1.99) donde se afirma que "El director de la Real Academia [...] considera que el término eurolandia no es el más apropiado para hacer referencia al nuevo espacio monetario...'. He querido incluir a eurolandia en este grupo, en el que eurose une a una base de composición, y no a una palabra autónoma, con plena conciencia sobre su origen. Aunque la base -landia proceda del inglés land ('tierra, país'), y éste a su vez del germánico landam, en sincronía pertenecería al mismo grupo y tendría el mismo comportamiento que las bases cultas. Así para el DRAE -landia es un elem. compos. (elemento compositivo) que significa "sitio de", "lugar de": ZumoLANDIA, fotoLANDIA. 
relación similar a la que se produce entre el nombre y el adjetivo que lo complementa (eurodiputados, euroejército, euroescéptico: 'diputados' o 'ejército' 'de la Unión Europea' vs. 'escéptico ante la UE'). En la mayor parte de los compuestos de este grupo, el segmento primero de la palabra alude a la UE, y no a Europa ${ }^{15}$.

Este último subgrupo constituye sin duda el conjunto más abundante y con mayor vitalidad en nuestros días, pues constantemente está recibiendo la incorporación de nuevos miembros. Son además los ejemplos más recientes por lo que, si figuran en obras lexicográficas, lo hacen muchas veces en algunas del tipo "diccionario de palabras contemporáneas" o "de neologismos", lo que da cuenta de la novedad con que son percibidos. Por otro lado, hace unos cuantos años no se encontraban recogidos en los diccionarios generales, mientras que ahora son de obligada presencia en los mismos ${ }^{16}$.

Sobre este grupo vamos a centrarnos en este artículo, y a él se referirán nuestras apreciaciones si no indicamos expresamente algo en contra. Consideraciones que pasan en este momento por plantear una serie de puntos que contribuyan a aclarar lo que todavía no ha sido estudiado con detenimiento, y que quizá nos acerquen a establecer la pertinencia de centrar todo el asunto en la discusión sobre si se trata de un caso de derivación o de composición o, por el contrario, si conviene dejar esa controversia en un segundo plano de manera que se deduzca fácilmente de lo que ha de ser previo y principal: el análisis del procedimiento y sus resultados.

3.1. Euro-se adjunta normalmente a nombres o adjetivos, aunque hemos encontrado un caso reciente en que se une a un verbo, quizás forzando las posibilidades combinatorias de este elemento: "Blair se euroenreda", un resultado que el periodista consigue hábilmente al dejar sin deshacer la ambigüedad entre los significados 'Europa' y 'UE'.

15 No se puede olvidar, no obstante, que en los primeros compuestos de este tipo euroremitía a 'Europa', como sucedía en eurocomunismo, eurocomunista (aprox. 1975) o eurocentrismo (1982).

16 Véase la nota 7 de este artículo. 
3.2. La unión de este segmento no implica ningún tipo de transformación categorial de la unidad a la que se incorpora el formante euro-, con lo cual los resultados conservan la categoría gramatical de las bases, fundamentalmente nombres y adjetivos y, en menor medida, verbos: Parlamento-> Europarlamento; escéptico-> euroescéptico; enredarse-> euroenredarse.

3.3. Desde el punto de vista formal, el encuentro de euro- con la palabra preexistente no plantea ningún problema cuando ésta empieza por consonante o por cualquier vocal que no sea la-o- (euroasociación, euroejército, euroinfocentro, eurohipoteca, eurocomicios, encontramos en EURODICAUTOM $)^{17}$. Cuando debe unirse a esta vocal hemos observado la vacilación entre la unión sin más con repetición de la vocal (eurooptimismo) y la supresión de una de las vocales (en la misma fuente comunitaria hemos podido leer Eurodisea [euro + odisea] para denominar una 'vuelta a Europa de los jóvenes') ${ }^{18}$.

3.4. En estas formaciones los componentes suelen presentarse soldados gráficamente aunque sea la primera vez que convivan en esa forma, lo que posiblemente esté dando cuenta del asentamiento de ese procedimiento en la lengua. A pesar de ello, esporádicamente lo hemos encontrado escrito con guión, algo que resulta perfectamente comprensible en algunos ejemplos encontrados en EURODICAUTOM en 1998, en los que la unión se producía con formas abreviadas no suficientemente asentadas: "acuerdo de asociación Euro-Med", "EURO-RDSI", o "solicitud Euro-TCB", "Trans-Euro-Noche" (el nombre propio de un término ferroviario, con sus equivalentes en el alemán Trans-Euro-Nacht o el francés Trans-Euro-Nuit).

17 No hemos hallado ningún ejemplo en el que la primera letra de la palabra preexistente sea la vocal $u$-.

18 La tendencia del español es a suprimir las vocales dobles, como ha sucedido en épocas anteriores, y como sigue sucediendo ahora mismo en otros casos muy cercanos al que nos ocupa: observamos que se alude a los telespectadores, con lo que la vocal final de la primera unidad y la inicial de la segunda se funden en una sola - - -. En el francés puede verse una tendencia similar al fundirse dos vocales: al comenzar una película leemos que ha colaborado en su producción la fundación europea Eurimages. 
No tienen la misma justificación, sin embargo, los casos extraídos del periódico El Mundo en 1994, entre los que convive alguna forma con guión, como euro-periplo (20.1.1994, p. 22) con otras que tienen un grado de fijación similar pero que, quizá por falta de coherencia, son escritas indistintamente con o sin guión: eurocámara o eurodiputados vs. Euro-parlamentario (12.6.1994, p. 18). Algo similar ocurre en unos ejemplos de $A B C$ (17.12.95) donde junto a eurodiputado o euroburbuja coexisten euro-realidad y euro-mundo. Es posible que esta vacilación se deba a un tratamiento incoherente, aunque, como nos enseña la Historia de la lengua, es lógica en esos momentos en que un cambio lingüístico -como es la incorporación de un elemento nuevo- se encuentra en pleno desarrollo. El tiempo se encargará de convertir a una de esas soluciones en la definitiva.

3.5. Euro- surge del truncamiento de una palabra de tres o cuatro sílabas (Europa o europeo). En el truncamiento cae la parte de la palabra que es interpretada por los hablantes como un segmento reconocible (un afijo, una base de composición). Algo que no sucede en este caso, pues difícilmente podemos suponer que los hablantes interpreten como un afijo flexivo o derivativo, en definitiva, como ese "segmento reconocible", a la parte que cae, sea -pa,-peo o -pea, por lo cual tendremos que buscar otra explicación ${ }^{19}$.

Quizás deberíamos hablar entonces de un alomorfo para la formación de nuevas unidades especializado en la posición inicial, o de un

19 No ocurre lo mismo en casos que hemos puesto como paralelos a euro-. Dino-, petroo auto- surgen del truncamiento de dinosaurio, petróleo y automóvil respectivamente. En todos ellos, ejemplos típicos de composición a la manera culta, permanecen las bases de composición que aparecen en posición inicial, mientras que las finales caen (-saurio, -óleo, -móvil).

En el Diccionario de Lingüística de J. Dubois et al. (1983) s.v. prefijo se dice que "el truncamiento (abreviación sintagmática) puede llevar al prefijo a asumir la carga semántica de toda la unidad”. Los ejemplos que aduce son auto(móvil) o mini(falda). Aunque en estos casos resulta un tanto problemático llamar al formante inicial "prefijo", a favor de su afirmación concurren ejemplos como hiper(mercado) o ex(mujer), en los que sí se puede hablar claramente de prefijos que, tras el truncamiento, asumen toda la carga semántica de la unidad. 
simple acortamiento de la unidad en esa forma euro- . En ambos casos el segmento resultante coincidiría con la base que podría funcionar en la composición a la manera culta, y que habría surgido como fruto de la tendencia que tienen una gran parte de los segmentos iniciales de compuestos de distinto tipo a convertirse en bisílabos ${ }^{20}$.

3.6. Las denominaciones más frecuentes que se han aplicado a formantes como euro- han sido las de pseudoprefijo o prefijoide, posiblemente porque hay determinados aspectos que nos acercan a su consideración como prefijo, eso sí, un tanto atípico ${ }^{21}$ :

a) Su aparición únicamente en posición inicial de palabra, algo que por otra parte no es exclusivo de los prefijos, pues muchas de las consideradas tradicionalmente como bases de composición cultas sólo aparecen en esa posición ${ }^{22}$.

b) El que no produzca un cambio de categoría gramatical en la base con la que se une, como sucede con los prefijos, que mantienen la categoría de la base ${ }^{23}$. No creemos, sin embargo, que éste sea un argumento que deba esgrimirse para oponerlo a la composición, en cuyo estudio no usamos la transformación categorial como dato definitorio de la misma, sino únicamente como un rasgo que puede ayudarnos en la identificación del núcleo del compuesto.

20 Tendencia que puede observarse en compuestos como cabizbajo, tal como aparece señalado en Francisco García Lozano (1978) "Los compuestos de sustantivo + adjetivo del tipo pelirrojo", recogido en Soledad Varela (1993: 205-214).

21 S. Varela y J. Martín García (1999: 4997) excluyen a euro- de la nómina de prefijos por su combinatoria.

22 En la composición culta resulta mucho menos frecuente que una base ocupe indistintamente la posición inicial o final de palabra (caso de pterodáctilo/dactilografía; dermoblasto/equinodermo; filosofía/anglófilo; etc.), siendo lo más normal la especialización en una de las dos posiciones.

23 Sin embargo M. Correia (1989) considera a euro- un "prefixo recategorizador" en secuencias como "o sector eurodólar" o "cartâo eurocheque". Sin estar seguros de que nuestra percepción de esas producciones portuguesas sea la correcta, nos parece que el uso adjetivo del nombre se produce independientemente de su unión con euro-. 
c) Su imposibilidad de funcionar como forma libre, algo en lo que coincide con los afijos ${ }^{24}$. Sabemos, no obstante, que muchos temas de composición, y en concreto la mayoría de las bases cultas, no tienen tampoco autonomía léxica, lo que ha llevado a que en algunos momentos (aunque no tanto en la época actual) ese tipo de composición no haya sido considerado como tal ${ }^{25}$.

d) Su recursividad y su alta productividad en el momento actual ${ }^{26}$. Aunque también es verdad que otros segmentos de palabra (el lava- de lavavajillas, o el pati- de paticorto) dan lugar a muchas nuevas formaciones, y no por eso nos atrevemos a considerarlos como prefijos.

3.7. Otras características de euro- nos llevan a percibirlo como un tema de composición que funciona dentro del procedimiento de composición a la manera culta:

24 De la misma manera que no nos está confundiendo el que un viento se llame euro, tampoco debe ofuscarnos el que la misma forma haya adquirido un nuevo uso al convertirse en una forma libre como denominación oficial de la moneda de la UE. Otro caso distinto, esporádico desde nuestro punto de vista, es el que aparece reflejado en el Diccionario del Español Actual de M. Seco et al.: un euro adjetivo, forma libre, que es definido como 'eurocomunista'. Los textos que recogen esa forma nos muestran efectivamente ese uso adjetivo y su procedencia de la abreviación de eurocomunista. Nos sorprende sin embargo que esté recogido este uso que no ha tenido apenas trascendencia, y que no aparezca el nombre de euro ('moneda'), pues como sabemos estamos ante un diccionario aparecido en 1999 que, según su título, recoge el español de ese momento.

25 La opinión que aparece reflejada en Alvar Ezquerra no es en estos momentos la más compartida; para él "en la composición participan dos o más unidades léxicas que pueden aparecer libres en la lengua" (1995: 20), con lo que alejaría de la composición a formas como cabizbajo o cariacontecido. Siendo coherente con su propio criterio clasifica todos los prefijos cultos dentro de la derivación con lo que se producen no pocas contradicciones (p. 49). Se acercan mucho más a nuestra opinión los criterios expuestos en Cabré (1994).

26 Para Mitterand (1963: 59) el aumento de su productividad es una característica que puede llevar a considerar estos elementos como afijos. También Peytard (1964), refiriéndose al caso de télé-, señala que ese rasgo es el que le permite afirmar que se encuentra ante un verdadero prefijo del francés. A esta última opinión se une Correia (1989) para otorgar a euro- la categoría de prefijo. 
a) Los hablantes lo reconocen como un tema de composición, al igual que sucede con otros muchos formantes con los que eurocomparte un buen número de características: ciber-, eco-, tele-, auto-, petro-, etc. Ello es debido probablemente a que se interpreta como una unidad que no es autónoma, pero que posee contenido léxico, al igual que otras bases de composición ${ }^{27}$.

b) A pesar de su diferente procedencia, su comportamiento es el mismo que el de un tema de composición culta que genera nuevos compuestos en la lengua al unirse a palabras ya existentes, relacionándose con la otra base de la misma manera que lo hacen otros temas reconocidos plenamente como cultos.

c) Asimismo, la - o de su terminación es interpretada como la vocal de unión que tienen los temas procedentes del griego o los temas patrimoniales que se han generado para formar compuestos mediante ese procedimiento: es lo que ha llevado a formar un compuesto -híbrido desde tantos puntos de vista-como rockódromo (rock[\&roll]+o+dromo, 'lugar donde se celebran grandes conciertos de rock'), o a que Victoria Abril pueda asegurar "los sexoadictos somos un montón" (El País, 12.2.99), generando un compuesto a la manera culta que el periodista no duda en reproducir, eso sí, utilizando la cursiva para dar cuenta de la novedad de esa formación ${ }^{28}$.

3.8. Puede interpretarse, y es posible que la realidad se corresponda con esta visión, que las primeras formaciones con euro- no se generaron en español sino en otra lengua, posiblemente inglés o francés. Algo que por otra parte carece de interés en la descripción de un procedi-

27 En esa línea se pronuncia M.T. Cabré (1994). Por su parte L. Bauer (1983: 215) comenta a propósito de las ICFs (inicial combining forms) que tienen mayor densidad de información léxica que los prefijos, aunque a veces sea difícil diferenciarlas de ellos.

28 A esa -o final se refiere L. Bauer (1983: 214) como un elemento que toman necesariamente determinadas formas para convertirse en ICFs combinables; el ejemplo que aduce es jazzophile. 
miento para la formación de nuevas palabras que responde perfectamente a los mecanismos de que el español dispone en la actualidad, tanto si se trata de derivación como de composición. Si las primeras formaciones fueron préstamos, llegaron de una manera tan natural al español que esta lengua pudo asumirlos pues no violentaban sus reglas morfonológicas, y se inscribían además dentro de un procedimiento activo que dio y sigue dando lugar a nuevas unidades, plenamente españolas, pero que coinciden en su mecanismo de formación con las que se dan en otras lenguas románicas o en inglés.

3.9. A pesar de los lazos indudables de este tipo de formantes con los prefijos, y de la dificultad que supone en muchos momentos el delimitar el terreno que respectivamente les corresponde, no parece necesario, y por tanto tampoco aconsejable, plantear la existencia de una categoría intermedia entre la composición y la prefijación, antes bien, los argumentos esgrimidos en los apartados precedentes ( 3.6 y 3.7 ) nos permiten decidirnos por considerar el procedimiento que estamos tratando como de composición a la manera culta, por lo cual a partir de estos momentos llamaremos a las formaciones de este tercer apartado compuestos subordinantes en los que euro- es una base de composición con las peculiaridades que acabamos de comentar y que no conviene olvidar: especialización en posición inicial, tema de composición sin autonomía léxica, recursividad y alta productividad en la actualidad.

\section{ENTRE EL SIGNIFICADO Y EL USO (Y EL ABUSO)}

Cuando en el apartado anterior hemos procedido a clasificar los ejemplos encontrados en tres grupos, nos hemos servido de un criterio fundamental como es la estructura interna del compuesto, algo que, como bien sabemos, está muy ligado a su significado.

Desde este punto de vista es necesario distinguir también varios grupos que sólo en parte coinciden con los anteriores.

Al primer grupo pertenecerían los compuestos coordinados del tipo euroasiático. Se caracterizan, como dice É. Benveniste (1977: 149), por su "nexo asindético", con lo que su significado puede parafrasearse 
por el del par de elementos que los forman unidos por la conjunción que los pone en posición de igualdad: 'europeo y asiático'. En todos los casos el significado del euro- de este grupo es el de 'europeo' o 'referido a Europa', haciendo referencia bien a todo el continente, o sólo a una parte de él ${ }^{29}$.

Ese mismo significado adjetivo tiene euro- en las formaciones subordinantes más antiguas de los otros dos grupos. Así, Eurovisión es la 'televisión europea', de la misma manera que eurocomunismo es el 'comunismo de los partidos comunistas europeos que no pertenecían a los países del Este', o Eurocopa es la 'competición futbolística en la que participan equipos de todos los países europeos ${ }^{30}$.

El aumento cada vez mayor de voces que incorporaban euro- pero que tenían un sentido restrictivo, haciendo referencia sólo a una parte del continente, ha llevado a que prácticamente todas las unidades aparecidas en los últimos años aludan con ese formante únicamente a una parte de Europa: la que integran los países de la Unión Europea. Y de hecho, cuando alguna de esas voces puede interpretarse como referida al todo, encierra en sí la suficiente ambigüedad como para poder entenderse también como alusiva a la Unión Europea. Es el caso del eurotúnel que une Gran Bretaña con el continente europeo, pero que a la vez es una obra profundamente comunitaria ${ }^{31}$.

29 Resulta conflictivo, no obstante, el decidirse a veces por el significado exacto de la voz, ya que ésta puede tener más de una interpretación. En "Barcelona reunirá la cumbre euromediterránea que debe consagrar la nueva política de asociación con los países de la otra ribera..." (El País, 21.1.1995, p. 4) se plantea uno de esos casos conflictivos: aquí no se hace referencia a todos los países europeos y mediterráneos, sino a los que además de esas dos condiciones tienen la de pertenecer a la Unión Europea. El hecho de que se trate de dos adjetivos de lugar es el que nos hace decidirnos más por su estructura de compuesto en el que se da una relación de coordinación entre las dos bases (europea y mediterránea).

30 La concepción a veces un tanto extraña de la realidad podría llevarnos a pensar en la posible incorrección de nuestra definición, o incluso en que euro- esté mal utilizado, ya que en esta competición participan también los campeones de países como Turquía o Israel.

31 Sobre la ambigüedad se construye también un anuncio encontrado en los hipermer- 
Como ya hemos indicado en el apartado correspondiente, estas formaciones subordinantes responden a la estructura complemento $+n u ́-$ cleo, por lo que el primer elemento (euro-) dice algo del segundo: restringe su significado concretándolo en esa parte de Europa llamada Unión Europea (UE), aunque de distintas maneras dependiendo fundamentalmente de la naturaleza gramatical y semántica de la base a la que se une. Es lógico por tanto que los ejemplos encontrados deban parafrasearse de formas diferentes:

a) Adj. + N. 'Institución, lugar, hecho, etc., que pertenece a la UE, forma parte de la UE, o está relacionado con ella'. Posee un sentido locativo: europarlamento, eurocámara, euroelecciones, euroejército, etc.

b) Adj. + Adj. 'Que tiene la característica señalada en el $2^{\circ}$ formante a causa de o ante lo europeo (de la UE)': europesimista, euroescéptico, etc.

c) Adj. + V. 'Que se produce la acción expresada por el verbo ante lo europeo (de la UE)' parecía ser el significado en el único ejemplo que habíamos encontrado, euroenredarse, pero cambió totalmente en el otro ejemplo, euronormalizar, posiblemente a causa de la diferente estructura argumental del verbo.

Si nos centramos en este grupo, el que más ejemplos ha proporcionado, como ya hemos apuntado y como puede verse en el anexo final, podemos observar que el grado de lexicalización no es el mismo en todos los casos encontrados. La "débil conexión entre el afijo y la base" a que se refería M. F. Lang desde su perspectiva de considerar estas formaciones como derivados (1990: 239), ha de ser entendida en los momentos actuales con reservas. Pocos hablantes españoles percibirían hoy como neologismos voces como eurodiputado o euroelecciones,

cados Champion a finales de 1999: Euroahorro, en el que no se sabe bien si nos encontramos ante un nuevo significado del elemento euro-, ya que el mensaje escrito estaba adornado con euros (monedas ) en segundo plano; ¿ ahorro en euros o uso connotativo de euro- sin más? Incógnita en la que descansa precisamente la fuerza de ese mensaje publicitario. 
tendrían más reparos en considerar como asentadas a euroejército o europesimismo, y probablemente otorgarían la etiqueta de novedosas a euronormalizar o europuzzle. Sin embargo esa percepción habría sido distinta unos años atrás, lo cual podría verse explicado fácilmente por ese factor tan relacionado con los cambios lingüísticos como es el paso del tiempo. Un hecho que se ve además respaldado por la particular evolución de la realidad.

La progresiva incorporación de España a las estructuras de la Comunidad Europea provocó el surgimiento de voces con euro- para denominar a esa nueva realidad. Actualmente es el propio asentamiento de la Unión Europea como una unión de estados en la que cada vez hay más elementos en común, más organismos o instituciones, más labores conjuntas, el que genera muchas de las unidades que en estos últimos años hemos visto nacer. Son por tanto las circunstancias las que provocan ese surgimiento, las que determinan también la pervivencia de estas voces.

H. Mitterand (1992: 58) dice que estos neologismos, "nacidos de las circunstancias desaparecen con ellas". Con las circunstancias nació el que un periodista ocurrente llamó Euroalbacete, equipo de fútbol de la localidad manchega que podría tener ese nombre tras la sentencia del caso Bosman, por la cual los jugadores de la UE podrían jugar al igual que cualquier natural del país en cuestión, con lo que el Albacete, como sucede ahora mismo con otros muchos clubes de fútbol españoles, podría verse repleto de jugadores procedentes de los países de la UE. Posiblemente hoy ya nadie hable del Euroalbacete, de la misma manera que nos hemos olvidado de aquellas otras unidades que fueron también flores de un día, los euromarcos o las europesetas (ver Anexo).

Nos encontramos pues ante la convivencia de unidades léxicas, especialmente las relacionadas con la política comunitaria, que tienen un grado de fijación cada vez mayor, con otras que cumplen en su momento una función denominativa -basada en la necesidad unas veces, en la arbitrariedad otras- pero que tienen una vida muy limitada. El problema que se nos plantea en estos últimos casos es saber si el formante euro- está aportando únicamente unos rasgos significativos determinados, o si además añade algún tipo de connotación positiva con- 
dicionada por el valor específico que la sociedad ha concedido a lo europeo en general y a lo comunitario en particular en los últimos años ${ }^{32}$.

Esa valoración ha generado una especie de moda en cuyo primer estadio se hallarían esas denominaciones innecesarias y poco elegantes en las que puede observarse una pobre habilidad en la construcción del compuesto (euro-realidad, euro-mundo, eurotrabajo). Su punto culminante estaría constituido por el grupo que podría ser etiquetado como de "extrapolación hortera o kitsch del formante": una serie de voces en las que euro- tiene un uso puramente connotativo. No existe ningún valor referencial en el euro- de formaciones como Eurocalzados o Eurotormes. No tienen ninguna relación con Europa o la Unión Europea las Eurofunerarias que encontramos en una calle de Sevilla, el eurosalmón de un hipermercado salmantino, o el eurosucre escrito en el envoltorio de un azucarillo con que endulzamos un café en París.

Puede que las causas de esta moda tengamos que buscarlas en el prestigio que para el hablante medio tienen lo europeo y quienes trabajan en los organismos comunitarios. Ellos parecen haber sido los primeros productores de estas voces tanto en español como en otras lenguas en las que posiblemente se generaron esas formas en primer lugar. Son entre otras las unidades con euro- las que constituyen lo que con buena dosis de humor algunos traductores de la Unión Europea en Luxemburgo denominan eurolalia, o a lo que R. Goffin (1994) llamaba eurolecte ${ }^{33}$.

32 Con la presuposición de esa valoración positiva contaban los patrocinadores del gran parque de atracciones europeo de Walt Disney al que llamaron Eurodisney. Sin embargo sorprende que en este caso la construcción con euro- no funcionase, y el nombre primero tuviera que ser sustituido al poco tiempo por el de Disneyland París.

33 Cuando Goffin habla en su artículo del eurolecte (el lenguaje utilizado en los escritos comunitarios), se refiere también a las eurolexies; hablando de los neologismos de forma como uno de los mecanismos lexicogenéticos para la formación del eurolecto, dice"... ou par le préfixe euro, qui se montre d'une belle vitalité dans eurobromètre, euroguichets, euroguide, eurolaser, euromanager, europartenariat, europêche, europessimisme, et euroforteresse, ou encore eurocommunisme ou eurodollar" (1994: 639). 
Son voces que aparecen fundamentalmente en textos informativos, producidos por personas cultas que generan el neologismo la mayor parte de las veces no por verdadera necesidad, pues la paráfrasis podría cumplir perfectamente la función denominativa que impone el surgimiento de una nueva realidad. Voces que aparecerán más adelante en otros textos informativos, los periodísticos, que difunden y comentan las noticias en torno a los asuntos comunitarios; textos producidos también por personas cultas que demuestran a veces su habilidad para generar la voz impactante que puede impresionar en un titular ("Blair se euroenreda"), pero que en otras ocasiones denotan una escasa preocupación por su lengua unida a una gran pobreza de recursos lingüísticos, lo que les lleva a dejarse arrastrar por una moda que no va a ayudarles a conseguir esa frescura que normalmente le pedimos a un texto correctamente producido ${ }^{34}$.

Dos son las consecuencias que se pueden deducir fácilmente del fenómeno anterior: en primer lugar, que el uso cada vez más frecuente de voces con ese formante puede conducir al abuso, como hemos visto en esos casos descontextualizados en los que euro- carecía de significado referencial; en segundo lugar, que el fenómeno que intentamos retratar con los ejemplos de euro- no es exclusivo de las voces que incluyen este formante.

A nada que ampliemos nuestros horizontes (y lo hemos estado haciendo durante todo el tiempo que nos ha llevado la investigación para la confección de estas notas) podemos observar que lo que se está produciendo con euro- ha ocurrido y sigue ocurriendo con otros formantes. Hemos podido ver la convivencia de impuestos ecológicos con ecotasas en un mismo texto, y después la sustitución del primero de ellos por el último a pesar de su más que dudosa corrección. Hemos encontrado bioarmas junto a armas biológicas, biotecnología y bioterroris-

34 El humorista Forges ridiculiza lo exagerado de esa moda en un chiste gráfico ( $E l$ País, 6.5.98). Un médico un tanto inexpresivo dice al paciente: "De su analítica se deduce que tiene altos los eurojilipollitos... y luego lo que es la tontolculinasa, dispará". A lo que el paciente, alegre a pesar de no haber entendido nada, responde: "Ouch!... what fine!". 
mo compartiendo un párrafo con agentes biológicos. Hemos constatado el uso y el posterior decaimiento de dinomanía en un determinado momento, y el abuso consiguiente con dinocuadernos, dinocepillos, dinodentrífico, dinopijamas y dinoedredones. Durante este tiempo hemos visto como empezábamos a codearnos con telenautas, videoguerras, tecnoindustrias, etnobombas o ciberpolicías. Y con todo ello nos hemos podido dar cuenta de que el uso prestigiado, la moda (que a veces conduce al abuso), no se centra en realidad en uno o varios elementos compositivos concretos, sino en el procedimiento -común a todos ellos- que da lugar a esos nuevos compuestos formados a la manera culta por el añadido de un nuevo elemento compositivo patrimonial a una palabra ya existente.

\section{A MODO DE CONCLUSIONES}

La observación a que hemos sometido a todas estas unidades a lo largo de este tiempo nos ha permitido comprobar una vez más la extraordinaria complejidad de los hechos lingüísticos, sea cual sea el nivel de análisis en que nos movamos. De hecho el estudio de esta cuestión morfológica menor nos lleva a pensar de nuevo en la necesidad de relativizar cualquier afirmación que se haga sobre el entramado del lenguaje en general, o sobre un aspecto concreto de una de sus manifestaciones.

El análisis de un procedimiento completamente vivo en estos momentos no hace sino resaltar el carácter provisional de nuestras consideraciones, basadas en un proceso todavía sin completar. No queremos aventurar el futuro, pero sí podemos hablar del pasado e intentar reflejar la situación de un presente que, también en este caso, constantemente va cambiando ${ }^{35}$.

35 De hecho, cuando ya habíamos decidido entregar este artículo a la imprenta nos encontramos con un nuevo ejemplo en el que, forzando las posibilidades de euro-, se le confiere un nuevo significado: 'moneda europea': "práctica calculadora de bolsillo, euroconversora" (anuncio aparecido en Phytoma, Revista profesional de sanidad vegetal, agosto/septiembre de 1999). 
Nos hemos encontrado con un problema conceptual que hemos tratado de aclarar considerando todos los elementos que confluían en él, y hemos podido observar cómo las dificultades en la delimitación de los conceptos se veían reflejadas en las diferentes denominaciones con que se aludía a un mismo concepto. La existencia de esa confusión terminológica nos obliga a plantear y apoyar una denominación -elemento compositivo prefijal-a la que consideramos coherente por varias razones $^{36}$ :

a) porque se trata de una denominación transparente y motivada. Desde nuestra perspectiva, nos hallamos ante un procedimiento de composición a la manera culta, por lo que en primer lugar hay que dar cuenta de que se trata de composición y de que, además, según una indicación que es sobre todo posicional, euro- precede siempre a la palabra preexistente a la que se adjunta;

b) porque se inscribe dentro de la tradición lexicográfica del español, especialmente el núcleo del compuesto ${ }^{37}$;

c) porque es una denominación perfectamente insertable dentro de una estructura denominativa que con coherencia refleje ese concepto y junto a él la posibilidad de que existan elementos que se pueden añadir a la parte posterior de la palabra preexistente (elementos compositivos sufijales), u otros para los cuales haya que dar cuenta de su inserción dentro de un procedimiento derivativo (elementos derivativos prefijales y sufijales).

36 No figura entre nuestros intereses aumentar la polémica (y con ella la confusión) sobre la denominación de estos elementos y del procedimiento en que están enmarcados sumándonos a ella con una nueva propuesta denominativa (véase R. Almela Pérez 1999, especialmente pp. 154 y ss). Sí abogamos, no obstante, porque un único término dé cuenta convenientemente del concepto que representa.

37 Formante podría haber sido igualmente elegido como núcleo de este compuesto sintagmático, pero es una forma menos asentada en la tradición lexicográfica española, y no representa un avance en la denominación, por lo que la descartamos. Elemento compositivo es la denominación usada por el diccionario académico desde su edición de 1970, tanto para formas antepuestas (hidro-) como para las pospuestas (-algia). 
Hemos constatado el uso, pero también el abuso, la creación oportuna y la llevada a cabo con escasa habilidad, la motivada y la generada por el más simple mimetismo. Y ante ello no podemos sino plantear la necesidad de guiarse por ese a veces tan difícil equilibrio entre "el riesgo y el placer de elegir" que debe presidir la actuación de los hablantes (J. A. Pascual Rodríguez 1996). Ante un procedimiento de formación de palabras latente que las circunstancias de que hablaba $\mathrm{H}$. Mitterand pueden activar en cualquier momento, el hablante ha de estar atento y preparado para poder usarlo. Es el conocimiento del tipo de texto que quiere producir, de la situación comunicativa en que se halla inmerso, o de su intención, lo que le llevará a decidirse por la denominación desarrollada o por la forma compuesta a la manera culta, pues tanto una como otra están bien formadas, aunque no sean igualmente adecuadas en todos los casos.

Y si en el uso han de ser la mesura y el equilibrio los patrones que guíen al hablante, en el diccionario el lexicógrafo ha de actuar con la prudencia que le ha dictado siempre el sentido común, mostrando la realidad del uso y dando cuenta de los diferentes significados de ese elemento compositivo para de esa manera facilitar a los usuarios la posibilidad de la formación del compuesto. Habrá de incluir en un artículo independiente el elemento compositivo y señalar convenientemente separados los distintos casos en que éste se aplica, con ejemplos de uso reales. Al mismo tiempo, tendrá que reflejar como entradas las formas ya asentadas en el léxico respetando, como es lógico, los resultados proporcionados por el análisis del corpus textual, que posiblemente apuntarían a la conveniencia de incluir europarlamentario, pero desaconsejarían en el otro extremo el ocasional Euroalbacete o el jocoso eurojilipollitos.

\section{BIBLIOGRAFÍA}

Adelstein, A. (1996): "Banalización de términos con formantes de origen grecolatino", en Actas del V Simposio Iberoamericano de Terminología. Edición electrónica: http://www.unilat.org/dtil/MEXICO/.

Alba DE Diego, V. (1983): "Elementos prefijales y sufijales: ¿derivación o 
composición?", en Serta Philologica F. Lázaro Carreter. Vol. I: Estudios de lingüística y lengua literaria, Madrid, Cátedra, pp. 17-21.

— (1985): "En torno a la derivación”, en Philologica Hispaniensia in honorem Manuel Alvar, II, Madrid, Gredos, pp. 21-32.

Almela Pérez, R. (1999): Procedimientos de formación de palabras en español, Barcelona, Ariel.

Alvar EzQuerra, M. (1978): "Notas para el estudio del formante de palabras español foto-", Analecta Malacitana, 1, pp. 313-326.

(1994): Diccionario de voces de uso actual, Madrid, Arco Libros. (1995): La formación de palabras en español, Madrid, Arco Libros.

BAuer, L. (1983): English Word Formation, Cambridge, Cambridge University Press.

Benveniste, É. (1977): Problemas de lingüística general, II, México, Siglo XXI.

Bosque, I. y Demonte, V. (eds.) (1999): Gramática descriptiva de la lengua española, Madrid, Espasa Calpe.

CABré, M. T. (1994): A l'entorn de la paraula, Valencia, Universitat de València.

Casado Velarde, M. (1985): Tendencias en el léxico español actual, Madrid, Coloquio.

— (1999): "Otros procesos morfológicos: acortamientos, formación de siglas y acrónimos”, en I. Bosque y V. Demonte (eds.), Gramática descriptiva de la lengua española, pp. 5075- 5096.

Correia, M. (1989): “Euro-: um novo prefixo do português?”. Artículo inédito.

— (1990): "Pseudoprefixo: um conceito incómodo para o estudo da derivaçâo prefixal". Artículo inédito.

CRUZ PIÑOL, M.C. (1999): Espan-L, un foro de debate en la Internet sobre la lengua española. Edición electrónica: http://elies.rediris.es/elies1/ Goffin, R. (1994): “L'Eurolecte: oui, jargon communautaire: non”, Meta, XXXIX. 4, pp. 636-642.

Gómez TorRego, L. (1995a): El léxico español actual: uso y norma, Madrid, Arco Libros.

(1995b): “¿El euro o la euro?, ¿el macro o la macro?”, Español actual, 63, pp. 83-84.

LANG, M. F. (1992): Formación de palabras en español, Madrid, Cátedra.

MitTerand, H. (1992): Les mots français, Paris, P.U.F., $8^{\mathrm{a}}$ ed.

PASCUAl RodríGuez, J. A (1996): El placer y el riesgo de elegir: Sobre 
los recursos derivativos del español, Salamanca, Universidad de Salamanca.

PÉrez GonZÁlez, G. (1985): “Observaciones acerca del elemento compositivo auto-”, Anuario L/L, 16, pp. 309-317.

Pérez Lagos, M. F. (1985): “Un nuevo 'elemento compositivo' en el DRAE: radio-", Español Actual, 44, pp. 87-94.

Peytard, J. (1964): "Motivation et préfixation: remarques sur les mots construits avec l'élément téle-", Cahiers de Lexicologie, 1, pp. 37 44.

(1969): “De l'ambiguïté sémantique dans les lexies préfixées par auto-". Langue française, 4, pp. 88-107.

Romero GuAlda, M. V. (1976): "Acerca del elemento tele-", Thesaurus, 31, pp. 502-511.

VALLÉs, T. (1996): Qüestions i propostes sobre la productivitat en la formació de mots, Barcelona, Universitat Pompeu Fabra. Trabajo de doctorado inédito.

VAREla, S. (ed.) (1993): La formación de palabras, Madrid, Taurus.

VARELA, S. y J. MARTín GARCÍA (1999): "La prefijación”, en I. Bosque y

V. Demonte (eds.), Gramática descriptiva de la lengua española, III, pp. 4993-5040.

WARREN, B. (1990): “The importance of combining forms", en W. U. Dressler et al. (eds.), Contemporary Morphology, Berlin/New York, Mouton de Gruyter, pp. 111-132.

\section{ANEXO}

NóMINA DE EJEMPLOS ESTUDIADOS

La siguiente clasificación se ha realizado atendiendo en primer lugar a la estructura del compuesto, y en segundo lugar al significado del formante.

Si la palabra aparece recogida en alguno de los diccionarios del español más usados se indica a continuación del ejemplo con las siguientes abreviaturas: Drae (Diccionario de la Real Academia Española, $21^{\mathrm{a}}$ ed., Madrid, Espasa-Calpe, 1992), DuE (Diccionario de Uso del Español, María Moliner, 2a ed., Madrid, Gredos, 1998), GdLe (Gran 
Diccionario de la Lengua Española, Larousse-Planeta, 1996), Vox (Diccionario General Ilustrado de la Lengua Española, $1^{\text {a }}$ ed., Barcelona, Bibliograf, 1990), SalSan (Diccionario Salamanca de la Lengua Española, Santillana-Universidad de Salamanca, 1996), y DeA (Diccionario del Español Actual, Manuel Seco et al., Madrid, Aguilar, 1999). También se indica si se encuentra en el DvuA (Diccionario de voces de uso actual de Manual Alvar Ezquerra, Madrid, Arco Libros, 1994).

Únicamente adjuntamos definiciones y ejemplos de uso de las voces que no aparecen en ninguno de los diccionarios citados.

1. Compuestos coordinados:

(euro- = 'europeo')

eurasiático (DEA)

euroafricano (GDLE, Vox)

euroasiático (DraE, DuE, SalSan, GDLE,

Vox, DEA)

euromediterráneo. De Europa y de los países del Mediterráneo al mismo tiempo. "Barcelona reunirá la cumbre euromediterránea..." (El País, 21.1.95, p. 4). europolaco (DVUA) eurosiberiano (Vox)

2. Compuestos subordinantes:

euro- + tema de composición (normalmente culto) (euro- = 'de Europa' o 'de la UE')

eurocracia (DVUA, DEA)

eurócrata (Dgle, SalSan, Vox, Dvua, DEA)

eurolalia Jerga utilizada por los funcionarios de la UE (sin testimonios escritos, pero oído en múltiples ocasiones a traductores de la UE).

eurolecto Eurolalia (sin testimonios escritos, oído a traductores de la UE).
Eurolandia Conjunto de países de la UE que han adoptado el euro como moneda única "Temor a una crisis en Eurolandia" (titular de El País, 4.5.98, p. 64). "El director de la Real Academia Española [...] considera que el término eurolandia no es el más apropiado para hacer referencia al nuevo espacio monetario europeo" (El País, 15.1.99).

3. Compuestos subordinantes:

euro- + palabra actual.

3.1. euro- = 'de Europa'

euroaliado (GDLE)

eurobag (DVUA)

eurobono (GDLE, Vox)

eurocéntrico Que considera todos los hechos poniendo a Europa como centro del mundo. "... sentimiento de que debe dejar de ser un órgano eurocéntrico, ya que los problemas más intensos ocurren fuera de las fronteras europeas" (El País, 9.11 .99 , p. 7).

eurocentrismo "... se manifiesta reiteradamente un macizo eurocentrismo" (G. Araya "El diccionario de americanis- 
mos" LEA, IV, 137-150; p. 148), (GDLE, Vox, DeA)

eurocheque (Due, GdLe, Vox, DeA)

eurocomunismo (Drae, Due, GdLe, SalSan, Vox, DEA)

eurocomunista (Drae, Due, GdLe, SalSan, DEA)

euroconector (GDle, SalSan, Dvua, DEA)

Eurocopa Competición futbolística en la que participan los campeones de copa de los distintos países europeos ("La Eurocopa 96 se sortea hoy en Manchester". El País, 22.1.94, p. 36).

euroderecha (GDLE)

eurodivisa (Drae, GdLe, Vox, Dea) eurodólar (Due, GdLe, SalSan, Vox, DEA)

eurofestival (DEA)

euroizquierda (GDLE, DvUA, DEA)

euromercado (DEA)

euromisil (Due, Gdle, Vox, DeA)

eurosocialismo (DEA)

eurosocialista (DVUA, DEA)

eurotarifa Tarifa telefónica que paga el usuario por realizar cualquier llamada de un lugar a otro de Europa. "Eurotarifa única (30 $\mathrm{ptas} . / \mathrm{min})$. Una tarifa que se mantiene sea cual sea el punto al que usted llame de Europa o España" (Anuncio de Cetel-RSLCOM, aparecido en la prensa el 18.3.1999)

eurovisión (DRAE, GDLE, SalSan, Vox) eurovisivo (DEA)

3.2. euro- $=$ 'de la UE'

euroagencia (DvUA)

Euroalbacete Equipo de fútbol de la ciudad de Albacete integrado mayorita- riamente por jugadores de los países de la UE ("El Euroalbacete es posible", El País, 16.12.95, p. 51).

euroastronauta (DVUA)

eurobarómetro (DVUA)

eurobeca Beca de intercambio entre universidades de la UE ("Las eurobecas Erasmus ofrecen la posibilidad de ampliar estudios en el extranjero", Tribuna Universitaria, Salamanca, $\mathrm{n}^{\circ}$ 2, p. 6). euroburbuja "La euroburbuja o la pasión por el papel escrito" ( $A B C$, 17.12.95, p. 35).

Eurocámara (DvUA, DEA)

eurocaza Avión cazabombardero de fabricación europea. "Firma del Eurocaza en Torrejón" (titular de El País, 22.2.94, p. 22).

eurociudad (DVUA)

eurociudadano (GDLE, SalSan)

eurocódigo (DVUA)

eurocomicios Elecciones a representantes en las distintas instituciones de la UE (múltiples ejemplos en la prensa española durante los meses de mayo y junio de 1994, así como en épocas electorales posteriores).

eurocomisario (DVUA)

Eurocuerpo Cuerpo de Ejército Europeo. “... el Eurocuerpo está formado por divisiones blindadas..." (El País, 15.11.99).

eurodebate (DVUA)

eurodeporte Deporte que se practica en la UE. “¿Eurodeporte?” (titular de un editorial de El País, 14.12.98).

eurodepósito (DVUA) eurodiputado (Drae, Due, GdLe, SalSan, Vox, DEA)

eurodocumento ".. se redactan febril- 
mente eurodocumentos" $(A B C, 17.12 .95$, p. 35).

euroejército (DVUA)

euroelecciones (GDle, Dvua, DEA)

euroenredarse Enredarse y confundirse a causa de los asuntos relacionados con Europa y la UE. "Blair se euroenreda" (titular de El País, 27.10.97).

euroentusiasmo Entusiasmo ante los asuntos de la UE. "El 'euroescéptico' del equipo de Prodi se convierte al 'euroentusiasmo' (El País, 7.9.99).

euroentusiasta (DVUA)

euroescepticismo (DEA)

euroescéptico (DVUA, DEA)

euroesclerosis (DVUA)

euroeuskera Sin un significado referen-

cial claro. "Superándolos a todos, incluso a Loyo-Lino, que tiene un morrazo euroeuskera, se encuentra Rosa Aguilar" (Maruja Torres, El País, 3.6.99).

eurofanático (DVUA)

eurofederalista (DVUA)

eurofóbico (DVUA)

eurofuncionario (DVUA)

euro-habitante “... esa burbuja de eurorealidad en la que vive el eurohabitante" (ABC, 17.12.95, p. 35).

euroinformación (DVUA)

euroinversión Inversión en productos financieros de la UE. "¿Euroinversión? Le ayudaremos a dar el salto" (Anuncio de Citibank aparecido en la prensa el 5.3.99).

eurojilipollitos Sin significado referencial. "De su analítica se deduce que tiene altos los eurojilipollitos" (chiste gráfico de Forges, El País, 6.5.98).

euro-manifestación “... capacitado para recoger las euro-manifestaciones de los dirigentes españoles" $(A B C, 17.12 .95, \mathrm{p}$. $35)$.

euromanifestante Habitante de la UE que participa en una manifestación sobre algún asunto que concierne a la política común. "No sostengo que todos los euromanifestantes contra los bombardeos de la OTAN..." (El País, 4.4.99).

eurometamorfosis "Eurometamorfosis financiera" (titular de un artículo de Economía de Emilio Ontiveros, El País, 4.5.98, p. 65).

euromarco Moneda de la UE (para el supuesto usuario alemán). “... nada de euromarco o europeseta, como acariciaba inicialmente el gobierno de Bonn" ( $E l$ País, 16.12.95, p. 2).

euromercado (GDLE, DVUA, DEA)

euromisión (DVUA)

euromoneda Moneda de la UE ( $E l$ Mundo, 28.11.95; El País, 17.12.95, p. 16). euro-mundo "Una cosa es la realidad y otra la euro-realidad; una el mundo, y otra el euro-mundo" (ABC, 17.12.95, p. 35).

Euronavidades Navidades en las que los asuntos de la UE están en primera línea de actualidad (título de un artículo de Pedro Schwartz, El País, 30.12.95, p. 48).

Euronormalizar Normalizar basándose en patrones europeos "El escritor alemán, que afirmó que hay que euronormalizar la literatura, recordó sus viajes a España" (El País, 24.10.99, p. 39).

euronota (DvUA)

Europarlamento Parlamento de la UE. "El Europarlamento supera sus reticencias..." (El País, 14.12.95, p. 6).

europarlamentario (GDLE, DVUA, DEA) europeseta Moneda de la UE (para el supuesto usuario español). “... nada de 
euromarco o europeseta, como acariciaba inicialmente el gobierno de Bonn" ( $E l$ País, 16.12.95, p. 2).

europesimismo (DVUA, DEA)

europesimista Pesimista ante los asuntos relacionados con la UE. (Se ha visto escrito repetidamente en la prensa de los últimos años).

Europol Policía de la Unión Europea. "El desarrollo de la policía europea (Europol)" (ABC, 17,12.95, p. 32).

Eurooptimismo Optimismo ante los asuntos relacionados con la UE (Titular del Telediario de TVE, $2^{\text {a }}$ edición, 27.11.93).

europtimismo (DVUA).

europuzzle (DVUA)

euro-realidad "Una cosa es la realidad y otra la euro-realidad; una el mundo, y otra el euro-mundo" (ABC, 17.12.95, p. $35)$.

eurorrealista (DVUA)

eurorrebelde "despertando los demonios eurorrebeldes" (El País, 14.12.95, p. 5).

eurotrabajo Trabajo dentro de la UE. "Eurotrabajos para Empléate a fondo" (titular de El País, 3.2.99).

eurotúnel (DuE, GDle, Dvua, DeA)

3.3. valor connotativo

Los ejemplos recogidos a continuación no son más que una pequeña muestra de los muchos que pueden encontrarse pertenecientes a este apartado. Se trata de nombres propios que, al contrario que en los casos anteriores, no intentan remitir a ninguna realidad europea, sino que transmiten únicamente una connotación positiva, por el prestigio que tiene lo europeo en un amplio sector de la sociedad española:

Eurocalzados, Euromuebles, etc. Nombres de tiendas de Salamanca, pero que pueden serlo de cualquier otra ciudad española.

Euroconferencias (anuncio en $A B C$, 2.4.95, p. 40).

Eurodesa Nombre propio dado a un proyecto realizado en una dehesa salmantina (entre Salamanca y Zamora) y financiado por la UE.

Euro Fiscal Duero Producto financiero de Seguros Unión Duero (1999).

Eurofruit'98 Feria agraria de Sant Miquel (Lleida, septiembre de 1998).

Eurofunerarias Visto en una calle de Sevilla, 1994.

Eurostar "el velocísimo tren Eurostar" (El País, 7.5.94, p. 3).

Eurotormes Transportes Eurotormes (en una furgoneta salmantina).

Eurosalmón "Llévese a casa el auténtico salmón de Noruega, el eurosalmón (oído por los altavoces del hipermercado Simago en Salamanca (29.12.95). 